\title{
ON THE SEMIGROUPS OF FULLY INVARIANT IDEALS OF THE FREE GROUP ALGEBRA AND THE FREE ASSOCIATIVE ALGEBRA
}

\author{
SAMUEL M. VOVSI
}

(Communicated by Maurice Auslander)

\begin{abstract}
Let $R$ be an integral domain, $K$ its field of fractions, $F$ a free group. Let $I$ and $J$ be fully invariant (=verbal) ideals of the group algebra $K F$. We prove that over certain domains the equality $I J \cap R F=(I \cap R F)$ $\times(J \cap R F)$ need not be true. A similar result is valid for fully invariant ideals of the free associative algebra. This implies that the product of pure varieties of group representations over an integral domain need not be pure, that there exist pure nonprojective varieties of group representations and of associative algebras, and also answers some other questions raised in the literature.
\end{abstract}

\section{INTRODUCTION}

The present work was inspired by the following question. Let $R$ be an integral domain, $K$ its field of fractions, $F$ a free group, and let $I$ and $J$ be fully invariant (i.e., invariant under all endomorphisms of $F$ ) ideals of the group algebra $K F$. Is it true that

$$
I J \cap R F=(I \cap R F)(J \cap R F) ?
$$

This question is actually a reformulation, in group ring terms, of a problem on varieties of group representations raised by Plotkin in the early 1970s. Without going into details (which can be found in [PV, V2, V5]), recall that all varieties of group representations over a given commutative ring $K$ form a semigroup under a naturally defined multiplication. This semigroup is canonically antiisomorphic to the semigroup $\mathbb{V}(K)$ of verbal ideals of $K F$. If $K$ is a field, the abstract structure of this semigroup was described in [PG] (see also [BL]): $\mathbb{V}(K)$ is a free semigroup with 0 and 1 . However, for $K$ not a field the structure of $\mathbb{V}(K)$ is by no means well understood.

From now on $R$ is an integral domain and $K$ is its field of fractions. It is natural that the first step in the study of the semigroup $\mathbb{V}(R)$ is to understand the relationship between $\mathbb{V}(R)$ and $\mathbb{V}(K)$. Let $\theta: \mathbb{V}(K) \rightarrow \mathbb{V}(R)$ be the map defined by $I^{\theta}=I \cap R F$. A straightforward verification shows that $\theta$ is injective

Received by the editors July 17, 1991 and, in revised form, March 27, 1992; this paper was presented at the 868th Meeting of the AMS (Special Session "Rings and Representations"), Philadelphia, PA, October 12, 1991.

1991 Mathematics Subject Classification. Primary 16A06; Secondary 20 C99. 
and that $\operatorname{Im}(\theta)$ consists of all verbal ideals $J$ of $R F$ such that $R F / J$ is torsion-free as an $R$-module (these verbal ideals and the corresponding varieties of group representations are called pure). Our initial problem can now be stated as follows: Is $\theta$ a homomorphism of semigroups? There are other equivalent formulations of the same problem, for instance: Is the product of pure varieties also pure? All these formulations plus some related questions were repeatedly discussed in [P, V2, PV] and several other papers.

A positive solution of the problem for $R$ a Dedekind domain was obtained by the author [V2, Corollary 9.10]. This result was later generalized by Bergman [B] who proved that if $R$ is a Dedekind domain, then (1) is valid for every right ideal $I$ and every left ideal $J$ of $K F$; for an exposition of his result see [V5, $\S 4.3]$. However, the problem over an arbitrary domain has remained open for a rather long time. In $\S 2$ of this note we construct an example solving it in the negative.

Another problem of a similar character was posed in 1985 by $\mathrm{L}^{\prime} \mathrm{vov}$. Let $R\langle X\rangle$ and $K\langle X\rangle$ be the free associative algebras on an alphabet $X$ over $R$ and $K$ respectively, and let $I$ and $J$ be fully invariant ideals $(=T$-ideals $)$ of $K\langle X\rangle$. Is it true that

$$
I J \cap R\langle X\rangle=(I \cap R\langle X\rangle)(J \cap R\langle X\rangle) \text { ? }
$$

Again over a Dedekind domain the answer turned out to be positive (see, e.g., [V3]), but over an arbitrary domain this has remained unknown until the present. We show that the counterexample to (1) can literally be carried over to the free associative algebra, thus giving a counterexample to $\left(1^{\prime}\right)$ as well. Using these examples, in $\S 3$ we answer several questions on varieties of group representations and associative algebras which were previously raised in the literature.

\section{THE COUNTEREXAMPLE}

Let $X=\left\{x_{1}, x_{2}, \ldots\right\}$ be a set of free generators of the group $F$ and let $Z=$ $\left\{z_{1}, z_{2}, \ldots\right\}$ be any set of the same cardinality. Then for any commutative ring $R$ there is a natural embedding, going back to Magnus [M], of the free group algebra $R F$ into the algebra $R\langle\langle Z\rangle\rangle$ of formal power series in the variables $z_{1}, z_{2}, \cdots$. Under this embedding the free generators $x_{i}$ of $F$ are mapped into $1+z_{i}$; the rest is uniquely determined. We will systematically identify $R F$ with its image in $R\langle\langle Z\rangle\rangle$; thus every $u \in R F$ is uniquely presented as a series

$$
u=u_{(0)}+u_{(1)}+\cdots+u_{(n)}+\cdots
$$

where $u_{(n)}=\sum \lambda_{i_{1} \cdots i_{n}} z_{i_{1}} \cdots z_{i_{n}}$, the homogeneous component of degree $n$ of $u$, is a finite $R$-linear combination of monomials of degree $n$ in the $z_{i}$. In particular,

$$
\begin{gathered}
x_{i}=1+z_{i} \quad\left(\text { and so } z_{i}=x_{i}-1\right), \\
x_{i}^{-1}=1-z_{i}+z_{i}^{2}-z_{i}^{3}+\cdots .
\end{gathered}
$$

An ideal $I$ of $R F$ is called homogeneous if for any $u \in I$ its homogeneous components $u_{(n)}$ are contained in $I$. Furthermore, each $u_{(n)}$ is uniquely presented as a sum of multihomogeneous components of $u$ (i.e., homogeneous in each variable), and $I$ is called multihomogeneous if for any element from $I$ all its multihomogeneous components belong to $I$ as well. 
Denote by $\Delta$ the augmentation ideal of $R F$. It is well known that an element $u \in R F$ belongs to $\Delta^{n}$ if and only if, in the above notation, $u_{(0)}=u_{(1)}=\cdots=$ $u_{(n-1)}=0$. This implies, in particular, that if $I$ is an ideal of $K F$ such that $\Delta^{n} \supseteq I \supseteq \Delta^{n+1}$, then $I$ is homogeneous.

Recall finally that an ideal $I$ of $R F$ is called fully invariant (or verbal) if it is invariant under all endomorphisms of $R F$ which are induced by endomorphisms of the group $F$. In this paper we will usually use the shorter term "verbal".

Let $K=k(t)$ be the field of rational functions in a variable $t$ over a field $k$. Noting that $K$ is the field of fractions of its subring $R=k\left[t^{2}, t^{3}\right]$, we can now construct the desired counterexample.

Theorem 1. Let $k$ be a field of characteristic $\neq 2,3, K=k(t)$, and $R=$ $k\left[t^{2}, t^{3}\right]$. Take in the group algebra $K F$ two elements

$$
u=z_{1}^{2} z_{2}-t z_{2} z_{1}^{2}, \quad v=z_{1} z_{2} z_{3} z_{4}
$$

(where $z_{i}=x_{i}-1$ ), and denote by $I$ the verbal ideal they generate in $K F$. Then

$$
I^{2} \cap R F \neq(I \cap R F)^{2} .
$$

Proof. 1. Consider in $K F$ the element

$$
w=(t u)^{2}=t^{2} z_{1}^{2} z_{2} z_{1}^{2} z_{2}-t^{3} z_{1}^{2} z_{2}^{2} z_{1}^{2}-t^{3} z_{2} z_{1}^{4} z_{2}+t^{4} z_{2} z_{1}^{2} z_{2} z_{1}^{2} .
$$

It belongs to $I^{2}$, and since all its coefficients are in $R$, we have $w \in I^{2} \cap R F$. Thus it remains to show that

$$
w \notin(I \cap R F)^{2} .
$$

Assume the contrary: $w \in(I \cap R F)^{2}$. Then $w$ can be presented in the form

$$
w=u^{1} v^{1}+u^{2} v^{2}+\cdots+u^{n} v^{n}
$$

where $u^{i}, v^{i} \in I \cap R F$ (do not confuse the upper indices with exponents!). Now note that

$$
\Delta^{3} \supset I \supset \Delta^{4}
$$

where $\Delta$ is the augmentation ideal of $K F$. It follows that each of the elements $u^{i}, v^{i}$ has no homogeneous component of degree less than 3 . Noting that the element $w$ is homogeneous of degree 6 and using (5), we conclude that

$$
w=w_{(6)}=u_{(3)}^{1} v_{(3)}^{1}+\cdots+u_{(3)}^{n} v_{(3)}^{n} \text {. }
$$

By (6), I is a homogeneous ideal. Therefore, $u_{(3)}^{i}, v_{(3)}^{i} \in I$, and since these elements are also contained in $R F$, we have $u_{(3)}^{i}, v_{(3)}^{i} \in I \cap R F$. Therefore, we may assume that all the $u^{i}, v^{i}$ in (5) are homogeneous of degree 3 . Furthermore, applying to (5) the endomorphism $\varphi$ of $F$ defined by

$$
x_{i}^{\varphi}= \begin{cases}x_{i} & \text { if } \quad i=1,2, \\ 1 & \text { if } i>2,\end{cases}
$$

we see that all the $u^{i}, v^{i}$ may be assumed to involve only the variables $z_{1}$ and $z_{2}$. 
Thus every $u^{i}$ and $v^{i}$ is an $R$-linear combination of monomials

$$
z_{1}^{3}, \quad z_{1}^{2} z_{2}, \quad z_{1} z_{2} z_{1}, \quad z_{2} z_{1}^{2}, \quad z_{2}^{2} z_{1}, \quad z_{2} z_{1} z_{2}, \quad z_{1} z_{2}^{2}, \quad z_{2}^{3} .
$$

Analyzing (3), (5), and (7), we see that the product of two monomials from (7) may yield a monomial of $w$ only if these two monomials are both of degree 2 in $z_{1}$ and of degree 1 in $z_{2}$. It follows that $w=u_{*}^{1} v_{*}^{1}+\cdots+u_{*}^{n} v_{*}^{n}$ where $u_{*}^{i}$ and $v_{*}^{i}$ are respectively the multihomogeneous components of $u^{i}$ and $v^{i}$ of degree 2 in $z_{1}$ and of degree 1 in $z_{2}$. However, it is known that if $I$ is a homogeneous verbal ideal of $K F$ and $u$ is a homogeneous element from $I$ such that $\operatorname{deg} u<\operatorname{char} K$, then all multihomogeneous components of $u$ belong to $I$ (this fact was proved in [V4] over a field of characteristic zero, but the proof remains valid under our assumptions). Since $\operatorname{deg} u^{i}=\operatorname{deg} v^{i}=3$ and char $K>3$, we obtain that $u_{*}^{i}, v_{*}^{i} \in I$. Thus we have eventually proved that $w$ can be written in the form (5) where all $u^{i}, v^{i} \in I \cap R F$ involve only $z_{1}$ and $z_{2}$ and are homogeneous of degree 2 in $z_{1}$ and homogeneous of degree 1 in $z_{2}$.

Denote, for a moment, by $F_{0}$ the subgroup of $F$ generated by $x_{1}, x_{2}$, by $\Delta_{0}$ the augmentation ideal of $K F_{0}$, and by $I_{0}$ the verbal ideal of $K F_{0}$ generated by $u$ and $\Delta_{0}^{4}$. A standard for variety theory argument shows that $I_{0}=I \cap K F_{0}$. Combining this observation with the above, we see that the element $w$ (which belongs to $K F_{0}$ ) can be written in the form (5) where all the $u^{i}, v^{i}$ belong to $I_{0} \cap R F_{0}$. In other words, from now on we may (and will!) assume that $F$ is the free group of rank 2 with free generators $x_{1}, x_{2}, \Delta$ is the augmentation ideal of its group algebra $K F$, and $I$ is the verbal ideal of $K F$ generated by $u$ and $\Delta^{4}$.

2. Our next objective is to find a $K$-basis of $I$ modulo $\Delta^{4}$. First of all, we note that $\operatorname{dim}_{K}\left(K F / \Delta^{4}\right)=15$ because the 15 monomials

$$
1 ; \quad z_{1}, z_{2} ; \quad z_{1}^{2}, z_{1} z_{2}, z_{2} z_{1}, z_{2}^{2} ; \quad z_{1}^{2} z_{2}, z_{2}^{2} z_{1}, z_{1}^{3}, \ldots
$$

form a natural basis in $K F$ modulo $\Delta^{4}$. Second, let us show that $\operatorname{dim}_{K}(K F / I)$ $=9$. A direct proof of this fact would require long and rather boring calculations; instead we will deduce it from one result proved in [PG]. Let $V$ be a 9-dimensional vector space over $K$ with a basis $b_{1}, \ldots, b_{9}$, and let $G$ be a subgroup of $\mathrm{GL}_{9}(K)$ generated by two unitriangular matrices

$$
\begin{aligned}
& g_{1}=e+e_{12}+e_{24}+e_{37}+t e_{59}-(1+t) e_{68}+e_{78}, \\
& g_{2}=e+e_{13}+e_{26}+e_{35}+t e_{48}+e_{69}-(1+t) e_{79}
\end{aligned}
$$

(here $e$ is the identity matrix and $e_{i j}$ are the standard matrix units). Consider the natural representation $\rho=(V, G)$ of $G$ on $V$. It is easy to see that $V$ is generated by $b_{1}$ as a $K G$-module and so $\rho$ is a cyclic representation. Therefore if $\operatorname{Reg}_{K} F=(K F, F)$ is the regular representation of $F$, then there exists an obvious epimorphism of representations

$$
\phi:(K F, F) \rightarrow(V, G) .
$$

The representation $\rho=(V, G)$ was constructed and studied in [PG]. In particular, it was proved there that $\rho$ satisfies the identities (2). Hence $\phi$ can be factored through $K F / I$ giving an epimorphism of $K$-spaces $K F / I \rightarrow V$. It follows that $\operatorname{dim}_{K}(K F / I) \geq 9$. 
On the other hand, among the 15 monomials (8) only the first nine can be linearly independent modulo $I$. Indeed, denoting by $\equiv$ equality modulo $I$, we have

$$
z_{1}^{2} z_{2} \equiv t z_{2} z_{1}^{2} \quad \text { and } \quad z_{2}^{2} z_{1} \equiv t z_{1} z_{2}^{2}
$$

Applying to any of these identities the endomorphism $x_{1} \mapsto x_{2}$ of $F$, we obtain $z_{2}^{3} \equiv 0$ and so $z_{1}^{3} \equiv 0$. Further, applying to the latter the endomorphism $x_{1} \mapsto x_{1} x_{2} x_{1}$ of $F$, we obtain

$$
\begin{aligned}
0 & \equiv\left(x_{1} x_{2} x_{1}-1\right)^{3} \\
& =\left(2 z_{1}+z_{2}+z_{1} z_{2}+z_{1}^{2}+z_{2} z_{1}+z_{1} z_{2} z_{1}\right)^{3} \\
& =8 z_{1}^{3}+6 z_{1}^{2} z_{2}+6 z_{1} z_{2} z_{1}+6 z_{2} z_{1}^{2}+z_{2}^{3}+\text { higher degree terms } \\
& =6\left(z_{1}^{2} z_{2}+z_{1} z_{2} z_{1}+z_{2} z_{1}^{2}\right)+\text { higher degree terms. }
\end{aligned}
$$

Since $I$ is a homogeneous ideal, it follows that $6\left(z_{1}^{2} z_{2}+z_{1} z_{2} z_{1}+z_{2} z_{1}^{2}\right) \equiv 0$. Since char $K \neq 2,3$, we have

$$
z_{1}^{2} z_{2}+z_{1} z_{2} z_{1}+z_{2}^{2} z_{1} \equiv 0 \text {. }
$$

By (9) and (10),

$$
z_{1} z_{2} z_{1} \equiv-z_{1}^{2} z_{2}-z_{2} z_{1}^{2} \equiv-(1+t) z_{2} z_{1}^{2} .
$$

All these relations show that the first nine of the monomials (8) span $K F$ modulo $I$. Thus $\operatorname{dim}_{K}(K F / I)=9$.

Since $\operatorname{dim}_{K}\left(K F / \Delta^{4}\right)=15$, we conclude that $\operatorname{dim}_{K}\left(I / \Delta^{4}\right)=6$. Now it is easy to find a basis for $I$ modulo $\Delta^{4}$. In view of the above, the following six elements belong to $I$ :

$$
\begin{gathered}
z_{1}^{3}, \quad z_{2}^{3}, \quad z_{1}^{2} z_{2}-t z_{2} z_{1}^{2}, \quad z_{2}^{2} z_{1}-t z_{1} z_{2}^{2}, \\
z_{1} z_{2} z_{1}+(1+t) z_{2} z_{1}^{2}, \quad z_{2} z_{1} z_{2}+(1+t) z_{1} z_{2}^{2} .
\end{gathered}
$$

These elements are linearly independent modulo $\Delta^{4}$ and so they form a desired basis.

3. We return now to the element $w$. It was proved in step 1 that if $w \in$ $(I \cap R F)^{2}$, then $w$ can be presented in the form (5) where each of $u^{i}, v^{i}$ belongs to $I \cap R F$, is homogeneous of degree 2 in $z_{1}$, and is homogeneous of degree 1 in $z_{2}$. Since (11) is a basis of $I$ modulo $\Delta^{4}$, it follows that each of $u^{i}, v^{i}$ is a $K$-linear combination of two elements:

$$
z_{1}^{2} z_{2}-t z_{2} z_{1}^{2} \text { and } z_{1} z_{2} z_{1}+(1+t) z_{2} z_{1}^{2}
$$

Hence for each $i=1, \ldots, n$ there exist $a_{i}, b_{i}, c_{i}, d_{i} \in K$ such that

$$
\begin{aligned}
u^{i} & =a_{i}\left(z_{1}^{2} z_{2}-t z_{2} z_{1}^{2}\right)+b_{i}\left(z_{1} z_{2} z_{1}+(1+t) z_{2} z_{1}^{2}\right) \\
& =a_{i} z_{1}^{2} z_{2}+b_{i} z_{1} z_{2} z_{1}+\left(\left(b_{i}-a_{i}\right) t+b_{i}\right) z_{2} z_{1}^{2}, \\
v^{i} & =c_{i}\left(z_{1}^{2} z_{2}-t z_{2} z_{1}^{2}\right)+d_{i}\left(z_{1} z_{2} z_{1}+(1+t) z_{2} z_{1}^{2}\right) \\
& =c_{i} z_{1}^{2} z_{2}+d_{i} z_{1} z_{2} z_{1}+\left(\left(d_{i}-c_{i}\right) t+d_{i}\right) z_{2} z_{1}^{2} .
\end{aligned}
$$

Since $u^{i}, v^{i} \in R F$, the coefficients in (12) and (13) must belong to $R$. Therefore,

$$
a_{i}, b_{i}, c_{i}, d_{i},\left(b_{i}-a_{i}\right) t,\left(d_{i}-c_{i}\right) t \in R
$$


Further, expanding the products $u^{i} v^{i}$, we have

$$
\begin{gathered}
w=\sum_{i=1}^{n} u^{i} v^{i}=\sum_{i=1}^{n}\left[a_{i} z_{1}^{2} z_{2}+b_{i} z_{1} z_{2} z_{1}+\left(\left(b_{i}-a_{i}\right) t+b_{i}\right) z_{2} z_{1}^{2}\right] \\
\quad \times\left[c_{i} z_{1}^{2} z_{2}+d_{i} z_{1} z_{2} z_{1}+\left(\left(d_{i}-c_{i}\right) t+d_{i}\right) z_{2} z_{1}^{2}\right] \\
=\sum_{i=1}^{n}\left(a_{i} c_{i} z_{1}^{2} z_{2} z_{1}^{2} z_{2}+b_{i} c_{i} z_{1} z_{2} z_{1}^{3} z_{2}\right. \\
\left.\quad+a_{i} d_{i} z_{1}^{2} z_{2} z_{1} z_{2} z_{1}+b_{i} d_{i} z_{1} z_{2} z_{1}^{2} z_{2} z_{1}+w_{i}\right)
\end{gathered}
$$

where each $w_{i}$ is a linear combination of monomials different from $z_{1}^{2} z_{2} z_{1}^{2} z_{2}$, $z_{1} z_{2} z_{1}^{3} z_{2}, \quad z_{1}^{2} z_{2} z_{1} z_{2} z_{1}, z_{1} z_{2} z_{1}^{2} z_{2} z_{1}$. Comparing the coefficients at the last four monomials in (15) with those in (3), we conclude that

$$
\begin{aligned}
& a_{1} c_{1}+\cdots+a_{n} c_{n}=t^{2}, \\
& b_{1} c_{1}+\cdots+b_{n} c_{n}=0, \\
& a_{1} d_{1}+\cdots+a_{n} d_{n}=0, \\
& b_{1} d_{1}+\cdots+b_{n} d_{n}=0
\end{aligned}
$$

Subtracting (18) from (16) and (19) from (17), we obtain

$$
\begin{gathered}
a_{1}\left(c_{1}-d_{1}\right)+\cdots+a_{n}\left(c_{n}-d_{n}\right)=t^{2} \\
b_{1}\left(c_{1}-d_{1}\right)+\cdots+b_{n}\left(c_{n}-d_{n}\right)=0
\end{gathered}
$$

Now subtract (21) from (20):

$$
\left(a_{1}-b_{1}\right)\left(c_{1}-d_{1}\right)+\cdots+\left(a_{n}-b_{n}\right)\left(c_{n}-d_{n}\right)=t^{2} .
$$

By (14), $\left(a_{i}-b_{i}\right) t \in k\left[t^{2}, t^{3}\right]$ and $\left(c_{i}-d_{i}\right) t \in k\left[t^{2}, t^{3}\right]$. This means that for each $i$ the constant term of the polynomials $a_{i}$ and $b_{i}$ coincide; the same is true for $c_{i}$ and $d_{i}$. Since $a_{i}, b_{i}, c_{i}, d_{i} \in k\left[t^{2}, t^{3}\right]$, it follows that the degree of each polynomial $a_{i}-b_{i}$ and $c_{i}-d_{i}$ in $t$ is at least 2, and so (22) is impossible. This contradiction completes the proof.

A similar result is valid for free associative algebras. Recall that if $K\langle X\rangle$ is the free associative $K$-algebra on a set $X=\left\{x_{1}, x_{2}, \ldots\right\}$ of free variables, then its ideal $T$ is called fully invariant (or $T$-ideal) if it is invariant under all $K$-endomorphisms of $K\langle X\rangle$. Such ideals are in a natural one-to-one correspondence with varieties of associative $K$-algebras.

Theorem 2. Let $k$ be a field of characteristic $\neq 2,3, K=k(t)$, and $R=$ $k\left[t^{2}, t^{3}\right]$. If $T$ is the $T$-ideal of $K\langle X\rangle$ generated by $x_{1}^{2} x_{2}-t x_{2} x_{1}^{2}$ and $x_{1} x_{2} x_{3} x_{4}$, then

$$
T^{2} \cap R\langle X\rangle \neq(T \cap R\langle X\rangle)^{2} .
$$

The proof is virtually the same as that of Theorem 1 . The only slight difference is in step 2. To prove the inequality $\operatorname{dim}_{K}(K\langle X\rangle / T) \geq 9$, one cannot apply the result of $[\mathrm{PG}]$ because the latter deals specifically with identities of group representations. Instead one should use the formula $\operatorname{dim}_{K}(K F / I)=9$ 
which has been already established in the proof of Theorem 1 , and verify that $K F / I$ satisfies the identities $x_{1}^{2} x_{2}-t x_{2} x_{1}^{2}$ and $x_{1} x_{2} x_{3} x_{4}$ as a $K$-algebra. Since $K\langle X\rangle / T$ is the free algebra of rank $|X|$ in the variety defined by these identities, there exists an epimorphism $K\langle X\rangle / T \rightarrow K F / I$, whence the desired inequality follows.

\section{Corollaries}

1. Let $\mathscr{Z}$ be a variety of group representations over an integral domain $R$ and let $I=\operatorname{Id} \mathscr{X}$ be the corresponding verbal ideal of $R F$. The variety $\mathscr{X}$ is called pure (projective) if $R F / I$ is torsion-free (projective) as an $R$-module. Is every pure variety projective? This question has been open since the early 1970s; to answer it, we first note that the result of $\S 2$ can be reformulated as follows.

Corollary 1 (solution of Problem 8.5.2 from [P]). There exists an integral domain $R$ (for example, $R=k\left[t^{2}, t^{3}\right]$ ) such that the product of pure varieties of group representations over $R$ need noi be pure.

Proof. The assertion that the product of pure varieties over $R$ is pure means that if $I_{0}$ and $J_{0}$ are verbal ideals of $R F$ such that $R F / I_{0}$ and $R F / J_{0}$ are $R$-torsion-free, then $R F / I_{0} J_{0}$ is also $R$-torsion-free. The latter is equivalent to saying that (1) is true for any verbal ideals $I$ and $J$ of $K F$. But this is impossible in view of Theorem 1 .

Let $R$ be an integral domain whose existence is guaranteed by Corollary 1 , and assume that every pure variety over $R$ is projective. Let $\mathscr{X}$ and $\mathscr{Y}$ be arbitrary pure varieties, and let $\rho=\operatorname{Fr} \mathscr{X}$ and $\sigma=\operatorname{Fr} \mathscr{Y}$ be their free cyclic representations of countable rank. By assumption, the modules of these representations are projective over $R$. Hence, by the main result of [V1] (see also [V2, Theorem 6.2]),

$$
\operatorname{var}(\rho \nabla \sigma)=\mathscr{X} \mathscr{Y}
$$

where $\rho \nabla \sigma$ is the triangular product of $\rho$ and $\sigma$. Since the module of the representation $\rho \nabla \sigma$ is torsion-free over $R$, the variety generated by this representation is pure. Therefore, the product of arbitrary pure varieties over $R$ must be pure, which is in contradiction with Corollary 1 . Thus we have proved

Corollary 2 (solution of Problem 18.2.8 from [PV]). A pure variety of group representations over an integral domain need not be projective.

It is still unknown whether this is true over $\mathbb{Z}$ [P, Problem 15]. We repeat this problem in terms of group rings. Let $I$ be a verbal ideal of the free group ring $\mathbb{Z} F$. Is it true that if $\mathbb{Z F} / I$ is a torsion-free abelian group, then it is a free abelian group?

2. As was mentioned in $\S 1$, over a Dedekind domain our initial problem has a positive solution. This fact was proved in [V1] using the technique of triangular products. Namely, let as usual $R$ be a domain and let $K$ be its field of fractions. To each representation $\rho=(V, G)$ over $R$ there naturally corresponds a representation $\rho_{K}=(K \otimes V, G)$, also regarded as a representation over $R$. Theorem 9.8 in [V2] states that if $R$ is a Dedekind domain and $\rho$ and $\sigma$ are pure representations over $R$, then

$$
\operatorname{var}\left(\rho_{K} \nabla \sigma_{K}\right)=\operatorname{var} \rho \cdot \operatorname{var} \sigma .
$$


An immediate corollary is that for $R$ a Dedekind domain the product of pure varieties is pure; in other words, (1) is true.

Problem 9.14 in [V2] asks if (23) is valid over any domain. Now we see that it is not the case: otherwise the product of pure varieties would always be pure which is impossible in view of Corollary 1.

3. Our proofs of Corollaries 1 and 2 were entirely based on Theorem 1 and on [V2, Theorems 6.2 and 9.8]. Since these three theorems have natural analogs for associative algebras (Theorem 2 and [V3, Theorems 1 and 2] respectively), the proofs of the corollaries remain valid for varieties of algebras as well. In terms of $T$-ideals the corresponding results can be stated as follows.

Corollary 3. There exists an integral domain $R$ such that the free algebra $R\langle X\rangle$ contains a $T$-ideal $T$ with the following properties:

(i) $R\langle X\rangle / T$ is $R$-torsion-free while $R\langle X\rangle / T^{2}$ is not;

(ii) $R\langle X\rangle / T$ is not $R$-projective.

The results of this note may give the impression that the situation of the free group algebra is completely analogous to that of the free associative algebra, at least when the above problems are concerned. But it is not the case. For example, it was noted that it is still unknown whether Corollary 2 is valid over $\mathbb{Z}$. On the other hand, the corresponding question for $T$-ideals can be solved very easily. Indeed, let $\mathbb{Z}\langle X\rangle / T$ be a torsion-free abelian group for some $T$-ideal $T$. A standard argument with the use of the Vandermonde determinant shows that $T$ is a multihomogeneous ideal. Therefore, the factor-algebra $\mathbb{Z}\langle X\rangle / T$ is a direct sum of its multihomogeneous components. Each of these components is a finitely generated torsion-free abelian group and, hence, a free abelian group. Consequently $\mathbb{Z}\langle X\rangle / T$ is a free abelian group itself.

\section{ACKNOWLEDGMENT}

In conclusion, I would like to express my gratitude to George Bergman for valuable conversations and suggestions.

\section{REFERENCES}

[B] G. M. Bergman, Personal communication, January 1983.

[BL] G. M. Bergman and J. Lewin, The semigroup of ideals of a fir is (usually) free, J. London Math. Soc. (2) 11 (1975), 21-31.

[M] W. Magnus, Beziehungen zwischen Gruppen und Idealen in einem speziellen Ring, Math. Ann. 111 (1935), 259-280.

[P] B. I. Plotkin, Varieties of group representations, Uspekhi Mat. Nauk 32 (1977), no. 5, 3-68; English transl., Russian Math. Surveys 32 (1977), no. 5, 1-72.

[PG] B. I. Plotkin and A. S. Grinberg, On the semigroups of varieties associated with group representations, Sibirsk. Mat. Zh. 13 (1972), 841-858; English transl., Siberian Math. J. 13 (1972), 585-596.

[PV] B. I. Plotkin and S. M. Vovsi, Varieties of group representations: General theory, connections and applications, Zinātne, Rīga, 1983. (Russian)

[V1] S. M. Vovsi, Varieties and triangular products of projective group representations, Sibirsk. Mat. Zh. 21 (1980), 56-62; English transl., Siberian Math. J. 21 (1980), 358-363.

[V2] - Triangular products of group representations and their applications, Progr. in Math., vol. 17, Birkhäuser, Boston, MA, 1981. 
[V3] _ Triangular products and identities of certain associative algebras, Algebra i Logika 26 (1987), 27-35; English transl., Algebra and Logic 26 (1987), 18-24.

[V4] _ Homogeneous varieties of group representations, Sibirsk. Mat. Zh. 29 (1988), 35-47; English transl., Siberian Math. J. 29 (1988), 360-371.

[V5] _ Topics in Varieties of Group Representations, London Math Soc. Lecture Notes, vol. 163, Cambridge Univ. Press, Cambridge, 1991.

Department of Mathematics, Rutgers University, New Brunswick, New Jersey 08903 E-mail address: vovsi@math.rutgers.edu

Current address: Department of Mathematics and Statistics, Trenton State College, Trenton, New Jersey 08650

E-mail address: vorsi@chaos.trenton.edu 